\title{
Los criterios de evaluación del ELE. Análisis de sus índices y propiedades en los referentes curriculares actuales
}

\author{
Carmen Guillén Díaz y Ma Jesús Santos Maldonado \\ Universidad de Valladolid \\ Jaime Ibañez Quintana y Esther Sanz de la CAL \\ Universidad de Burgos
}

Recibido: 10 noviembre 2007 / Aceptado: 13 marzo 2008

ISSN: $1697-7467$

\begin{abstract}
RESUMEN: Ante la prioridad y necesidad de homogeneidad y coherencia en la evaluación de las competencias en lenguas para la homologación y transparencia en los diplomas y las certificaciones, los autores se remiten -por una parte- a los referentes curriculares actuales: el Marco común europeo de referencia para las lenguas: enseñanza, aprendizaje, evaluación (2002) y, por su carácter subsidiario y actualizado, el Plan Curricular del Instituto Cervantes (2006). Por otra parte, y con la finalidad de optimizar las acciones relativas a la evaluación del ELE en particular, elaboran un dispositivo cuya modelización exponen en sus dos niveles: concepción y aplicación. Dicho dispositivo -mediante los procedimientos y las estrategias propias del análisis de contenido como método de indagación- está orientado a proporcionar a los profesionales implicados un repertorio de criterios de evaluación, definidos de forma pertinente y utilitaria, que sirva de guía para la toma de decisiones en cada situación de enseñanza/aprendizaje del ELE.

Palabras clave: Criterios de evaluación, Marco común europeo de referencia para las lenguas, Plan curricular del Instituto Cervantes, Español lengua extranjera, análisis de contenido.
\end{abstract}

Evaluation criteria for Spanish as a foreign language. An analysis of indexes and properties in current curricula

\begin{abstract}
Bearing in mind the priority and the need of the homogeneity and coherence of the languages competences assessment in order to get the homologation and transparency of the diplomas and certification, the authors refer to the present curricula references: Common European Framework of Reference for Languages: Learning, Teaching, Assessment (2002) and Plan Curricular del Instituto Cervantes (2006) because of their subsidiary and updated character. On the other hand, the authors will create a devise which is defined into two different levels: conception and application, in order to optimize the actions on the SFL assessment. By means of the procedures and strategies of the content analysis as a research method, this device will give the professionals a repertoire of assessment criteria, defined in useful and relevant way as a guide in order to take decisions in each SFL learning /teaching situations.
\end{abstract}


Key words: Assessment criteria, Common European Framework of Reference for Languages, Learning, Teaching, Plan Curricular del Instituto Cervantes, Spanish as a Foreign Language, content analysis.

\section{Consideraciones previas}

El Marco común europeo de referencia para las lenguas: enseñanza, aprendizaje y evaluación, MCER (2002) ${ }^{1}$, resultado de los trabajos de los expertos de la División de Políticas Lingüísticas del Consejo de Europa, publicado y difundido en las versiones de 36 lenguas, se ha destinado a cumplir la finalidad sociopolítica y educativa para la que se elaboró ${ }^{2}$ de forma que, hoy, es operativamente un documento de referencia generalizada entre aquellos profesionales responsables en materia de enseñanza, aprendizaje y evaluación de lenguas ${ }^{3}$.

Este documento de vocación internacional, con el estatus de material curricular ${ }^{4}$, se está introduciendo con normalidad en la formación inicial y continua de profesores ${ }^{5}$. Se entiende que debe servir para:

- Concebir y elaborar los programas de lenguas en términos de competencias y niveles de competencia.

- Lograr la coherencia en el interior de los sistemas educativos y entre los sistemas educativos de los Estados miembros del Consejo de Europa (Guide pour l'élaboration des politiques linguistiqes éducatives en Europe, 2007: 106), desde la construcción de una «cultura conceptual y pedagógica común» (Guillén Díaz, 2005).

- Establecer las bases de una evaluación que asegure la coherencia, la transparencia y la homogeneidad en las certificaciones, cuyo reconocimiento revierte en el mundo laboral y favorece la movilidad profesional; y las de una auto-evaluación individual de las competencias en lenguas, a través de la aplicación del Portfolio europeo de lenguas $^{6}$, concebido para dar cuenta del seguimiento y la continuidad del aprendizaje de lenguas.

\footnotetext{
${ }^{1}$ Nos referimos a la edición impresa en español, tal y como se consigna en el apartado de bibliografía.

${ }^{2}$ La promoción y desarrollo del plurilingüismo y pluriculturalismo y la mejora de la calidad de la enseñanza de lenguas.

${ }^{3}$ El MCER aparece ya citado como referente en los programas oficiales, en el nuevo currículo establecido para las lenguas en los niveles de enseñanza obligatoria y postobligatoria: Reales Decretos que establecen las enseñanzas mínimas de la Educación Infantil, primaria y secundaria (B.O.E.del 4.1.2007; del 8.12.2006 y del 5.1.2007 respectivamente; en las Escuelas Oficiales de Idiomas, Centros y Servicios de lenguas de las Universidades, en los certificados lingüísticos del MEC (DELE), etc., y de forma más reciente en los Planes de acogida e integración de inmigrantes (Guillén, C,; Ibáñez, J; Santos, Mª J. y Sanz, E.,(2006). Igualmente se nos remite à él, para la evaluación de competencias en lenguas y la concepción de pruebas de examen (Milanovic, 2002).

${ }^{4}$ Cuya estructura y organización significativas se abordan ampliamente en García Doval, F.; Guillén, C.; González Piñeiro, M.; González Porto, J.; Serna, I. y Vez, J. M. (2004). Ver: Observatorio Atrium Linguarum: http://www.atriumlinguarum.org

${ }^{5}$ De forma concreta, en el seno de los objetivos del Instituto Superior de Formación del Profesorado, se han desarrollado acciones formativas cuyos contenidos han sido objeto de publicación. Para el objeto de estudio que nos ocupa remitimos al reciente volumen dirigido por Montes Mozo (dir)(2005).

${ }^{6}$ Ver en textos de referencia del Consejo de Europa - División de Políticas Lingüísticas http://www.coe.int/ portfolio/
} 
- Poner en relación las formas de evaluación y los conocimientos adquiridos para la competencia clave: Comunicación en lenguas extranjeras, desde cuya definición se nos remite al conjunto de conocimientos, aptitudes ${ }^{7}$ y actitudes que la configuran, tal y como se expresa en la Recommandation (2006/962/CE):

...elle s'appuie sur l'aptitude à comprendre, exprimer et interpréter des concepts, des pensées, des sentiments, des faits et des opinions, à la fois oralement et par écrit (écouter, parler, lire et écrire) dans diverses situations de la vie en société et de la vie culturelle $(\ldots .).(\ldots)$ demande aussi des compétences comme la médiation et la compréhension des autres cultures. Le degré de maîtrise variera selon les quatre dimensions concernées (écouter, parler, lire et écrire) et en fonction des langues, ainsi qu'en fonction du contexte social et culturel, de l'environnement, des besoins et/ou intérêts de chacun. (p. 14)

Todos estos aspectos convergen en la necesidad de definir los criterios de evaluación como elementos de información- en un sistema que nos permita decidir sobre la presencia o no de las cualidades que dan cuenta del grado de logro en las competencias en lenguas (Raynal y Rieunier, 1997).

En este orden de cosas, tal y como expresaba recientemente Patrick Riba ${ }^{8}$, el impacto más evidente del MCER (2002) ha sido el de las escalas de niveles, ante cuyas especificaciones entendemos que se requiere un gran esfuerzo de armonización en la terminología relativa a:

- Los elementos del aprendizaje de la competencia clave Comunicación en lenguas extranjeras, que conciernen a la definición de objetivos y a la categorización de contenidos de enseñanza /aprendizaje, así como a la pertinencia y utilidad de los criterios de evaluación.

- La enseñanza, que concierne a las actividades comunicativas de la lengua, a través de las que se evalúa esta competencia.

- La evaluación del grado de competencia en una lengua, que concierne a los criterios de evaluación.

Se trata de especificaciones en torno a los 6 niveles comunes de referencia según una escala global (A1, A2, B1, B2, C1, C2), mediante descriptores de competencias comunicativas de la lengua (MCER, 2002: Capítulo 5) y descriptores de actividades comunicativas de la lengua y de estrategias (MCER, 2002: Capítulo 4). Descriptores que al formular lo que el alumno y el usuario de una lengua «es capaz de...; puede...» en la perspectiva de una comunicación eficaz, se nos muestran, ante todo, como orientadores por excelencia de los objetivos, los contenidos y los criterios de evaluación, a la hora de concebir y elaborar los

\footnotetext{
${ }^{7}$ El término «aptitud» aparece utilizado, para el mismo concepto, al lado de «capacidad», «habilidad» o «destreza». Si bien, en muchos casos es empleado como sinónimo erróneamente (Legendre, 1993).

${ }^{8}$ Responsable del Bureau du DELF/DALF Centre International d'Etudes Pédagogiques (Sèvres), con motivo de las Jornadas de reflexión sobre la evaluación del francés, celebradas en Egipto (3-4 de marzo de 2007).
} 
programas de lenguas. De forma consecuente, se nos muestran pues como instrumento técnico para la elaboración de las pruebas o exámenes para lo cual, y completando el Capítulo 9 del MCER, podemos disponer del documento titulado Manuel pour relier les examens de langues au Cadre européen común de référence pour les langues.

\section{Problemática actual de la evaluación del ele}

Sucede que, en estos últimos años, estamos asistiendo a hechos evidentes y destacables tales como que la adopción del MCER (2002) no se limita ya solamente al contexto europeo en la enseñanza/aprendizaje de lenguas (caso, por ejemplo, de las instituciones de enseñanza superior de países latinoamericanos, al incorporar en sus planes de mejora el desarrollo de competencias comunicativas en lenguas extranjeras de sus titulados para los ámbitos profesionales $)^{9}$; que la enseñanza de lenguas extranjeras desde las primeras edades en los sistemas educativos nacionales se ha generalizado; que -en el caso particular que nos ocupa aquí- el incremento de la demanda de una enseñanza/aprendizaje del español como lengua segunda (L2) / lengua extranjera (ELE) se acompaña de una fuerte demanda de certificaciones de su dominio.

Se trata de hechos, entre otros, de los que se deriva -también de forma evidente- un incremento de los interrogantes planteados por parte de los profesores sobre las posibilidades de aplicabilidad global del MCER, y, de forma concreta, sobre la evaluación del ELE relativa a los niveles comunes de referencia, dado que esta evaluación:

- es uno de los actos profesionales de la responsabilidad más directa del profesor para quien, además, es un factor de influencia en la mejora de la calidad de los aprendizajes (Roy, 1991) que nos remite a la idea de «impacto de los diplomas de español» contemplada por Parrondo Rodríguez (2004: 979);

- está sometida a una fuerte presión institucional y social, sobre todo por las repercusiones que toda certificación tiene para el mundo del trabajo y la movilidad profesional y de otro orden;

- se espera que se efectúe realmente en función de los niveles comunes de referencia del MCER a los que, al respecto, se remiten los autores de la actualización del Plan curricular del Instituto Cervantes (2006: Capítulo 1). Documento recientemente elaborado como base en la preparación de los programas de enseñanza del español.

Y observamos que, en esta actualización del Plan curricular, se han contemplado los niveles organizados en torno al «alumno como sujeto de aprendizaje... agente social... hablante intercultural y... aprendiente autónomo», y en torno a «la lengua como objeto del aprendizaje» (Plan curricular del Instituto Cervantes, 2006: 31); siendo en la dimensión del

\footnotetext{
${ }^{9}$ Ver en una perspectiva más amplia al respecto http://www.oei.es/certel.htm/ que nos ofrece el documento de trabajo del Proyecto CERTEL (Las certificaciones de conocimientos de lenguas en los países iberoamericanos. Panorama actual y perspectivas). Podemos añadir, en este sentido, el caso de la reforma global de la enseñanza del japonés lengua extranjera, preparada por Japan Educational Exchanges and Services, para la que está sirviendo el modelo del MCER.
} 
alumno como agente social, en la que las acciones relativas a la enseñanza, el aprendizaje y la evaluación se centran en el conocimiento de los elementos del sistema de la lengua y en la capacidad para desenvolverse en las situaciones de comunicación social.

Desde estos planteamientos, se nos ofrecen objetivos y contenidos:

- Objetivos generales, acompañados cada uno de ellos de especificaciones que desglosan el enunciado general y que presentan características del aspecto que se trata ${ }^{10}$, así como una descripción general de cada uno de los niveles («...especificaciones diferentes para cada uno de los (dos) niveles que constituyen cada etapa ${ }^{11} \gg$ p. 78).

- Contenidos, categorizados como inventarios de «material lingüístico» y de «aspectos culturales» que se encuentran, respectivamente, en los Capítulos del 2 al 9 y del 10 al 12 .

Así es como los interrogantes planteados por el profesorado provienen de dos grandes incertidumbres prácticas:

A) La aplicación de una evaluación de los progresos en ELE que debe inscribirse en el enfoque adoptado, es decir, según la perspectiva accional derivada de la concepción sobre el aprendizaje y uso de las lenguas por la que se ha optado en el MCER (2002: Capítulo 2).

B) La identificación-interpretación de los criterios de evaluación en los documentos referenciales citados, por cuanto que éstos les están sirviendo de guía en las decisiones técnicas curriculares al respecto.

Estas incertidumbres están generadas a su vez por una serie de dificultades a cuyas manifestaciones, por parte de los profesores, hemos accedido en los lugares de formación inicial y continua.

Respecto a la aplicación de una evaluación del ELE según una perspectiva accional, sus dificultades entroncan con el hecho de que toda actividad de evaluación -como puntualizan Puren (2001) y Bourguignon, Delahaye et Puren (2007)- implica evitar que:

- se refiera a «saberes», a «conocimientos descontextualizados», sino más bien a «tareas» que pueden ser no solamente de orden lingüístico, y que se relacionan con un saber-hacer y un saber-ser en cada escenario o situación de comunicación debidamente contextualizada;

- se dirija solo a un alumno-estudiante de ELE, sino a un alumno-usuario de ELE (MCER, 2002: 9) de quien se espera que demuestre un grado determinado de pertinencia y eficacia en las tareas efectuadas en la lengua respecto de un objetivo final;

\footnotetext{
${ }^{10}$ Cada objetivo general -en la dimensión de agente social- se relaciona con un aspecto estimado relevante. A saber: las transacciones, las interacciones sociales y los textos orales y escritos a los que se confronta el alumno o usuario.

${ }^{11}$ En el Plan curricular del Instituto Cervantes (2006) se utilizan las denominaciones: etapa o estadio inicial -A1 y A2-; etapa o estadio avanzado - B1 y B2; y etapa o estadio superior - C1 y C2 para referirse, en correspondencia con el MCER, respectivamente a: usuario básico; usuario independiente; usuario competente. (p. 77).
} 
- se realice solamente en forma de ejercicios / cuestiones de opción múltiple;

- carezca de sentido, porque se pueda poner en relación objetivos y criterios de evaluación, siendo «analítica», es decir, aportando elementos cualitativos a los puntos atribuidos como nota para hacer consciente al alumno no solo de «lo que debe ser capaz de...» (función sumativa de la evaluación), sino también de «cómo, en un momento dado, es capaz de...» (función formativa), (Ketele, 1986; Delorme, 1994; Lussier et Turner, 1995)

Respecto a la identificación-interpretación de los criterios de evaluación, -y en función de los posibles tipos de evaluación ${ }^{12}$ realizados- las dificultades manifestadas por los profesores se refieren:

- por una parte, a la multiplicidad de escalas - los 6 niveles citados - y a la cantidad de descriptores $^{13}$ de competencias y actividades comunicativas de la lengua que, igualmente, dan lugar a una gran incertidumbre y a una cierta confusión; y

- por otra parte, a los criterios de evaluación que para muchos no son demasiado «legibles» en esos descriptores que propone el MCER o en esos objetivos generales del Plan curricular del Instituto Cervantes. Al mismo tiempo coinciden en que, como expresa Tagliante (2005), no todos los descriptores de competencias son directamente evaluables y no se especifican los contenidos de orden lingüístico u otros.

Ante estos factores y en la perspectiva de la resolución de problemas, entendemos que se necesita disponer de un conjunto de precisiones sobre los criterios de evaluación con la finalidad de:

- Contribuir a crear un consenso socio-profesional en torno a aquellos criterios de evaluación que, por sus índices y propiedades, constituyan una base cognoscitiva y un instrumento intelectual de referencia para el acto profesional de evaluación que nos ocupa, en cada contexto de enseñanza y aprendizaje del ELE (Guillén, Blasco, Calleja, 1998).

Lo cual pasa por preguntarse además, para determinar en última instancia ¿cuáles son válidos, apropiados y realmente evaluables?

\section{LOS CRITERIOS DE EVALUACIÓN EN LOS REFERENTES CURRICULARES COMO UN OBJETO DE ESTUDIO PERTINENTE Y ÚTIL: SU ANÁLISIS}

Al acceder a lo que aporta el MCER respecto a las exigencias y demandas socioinstitucionales, es evidente que se nos sitúa ante una nueva cultura de la evaluación más realista y fiable que requiere, a su vez, una reflexión mas precisa en su práctica para efectuar las necesarias interrelaciones e interdependencias entre:

\footnotetext{
1226 tipos que presenta y describe el MCER (2002) en el Capítulo 9, apartado 9.3., dando cuenta de las diferentes funciones de la evaluación.

${ }^{13}$ Sabemos que se cuenta con unos 500 descriptores elaborados por North, B. (1996).
} 
- La formulación de objetivos de aprendizaje y la determinación de contenidos.

- El diseño de actividades de enseñanza / aprendizaje y de actividades de evaluación.

- La emisión de un juicio de valor y el fundamento de esa decisión.

Así pues, proceder a reactualizar la reflexión sobre la evaluación, conducente a la elaboración de un conjunto de precisiones sobre los criterios de evaluación en función de los niveles comunes de referencia, se traduce para nosotros en la pertinencia ${ }^{14}$ de un estudio analítico de los dos documentos citados como referentes curriculares. Estudio que articulamos en torno al interrogante más específico formulado en los siguientes términos:

¿Qué hay que tener en cuenta de esos descriptores y de esos objetivos para construir la evaluación, en la idea de una armonización y comparabilidad de las calificaciones y certificaciones en lengua, buscando un equilibrio en las funciones de la evaluación?

Consecuentemente, centramos nuestra atención en las informaciones ineludibles de orden formal, axiológico, práxico y explicativo que nos proporcionan estos referentes curriculares, a través de dos objetos textuales complementarios en particular:

A) Los descriptores de las competencias comunicativas de la lengua junto a los de las actividades comunicativas de la lengua (MCER, 2002, Capítulos 5 y 4 respectivamente), ya que los componentes de las competencias se encuentran en las actividades comunicativas de la lengua y considerando además, que los descriptores de las competencias no serán directamente evaluables si no es a través de las actividades.

B) Las definiciones de objetivos generales (Plan curricular del Instituto Cervantes, 2006: Capítulo 1) que se acompañan de los textos que los desglosan en varios puntos (entre uno y tres) y de los que especifican sus características, así como de los que presentan las descripciones generales de cada nivel, ya que se nos indica que en ellas:

se pretende dar una información amplia, a grandes rasgos, que permita al usuario hacerse una idea suficientemente clara del alcance de los niveles definidos, pero sin detallar en exceso los aspectos que se identifican ni aportar un análisis técnico de las categorías que se necesitan para elaborar los objetivos más concretos de los programas de los cursos de español (p. 74).

\subsection{Objetivos de este estudio}

En este orden de cosas, hemos elaborado un dispositivo que -como sistema intelectual y técnico- nos permita:

a) Elaborar un repertorio de criterios de evaluación transparentes, comunes y constantes $o$ estables (propiedades ${ }^{15}$ ) -con carácter «transversal» a todas las actividades

\footnotetext{
${ }^{14}$ La pertinencia en el ámbito disciplinar de la Didáctica de las Lenguas-Culturas y en la formación de profesores se entiende como la conformidad de los conocimientos relativos a la evaluación a los que podemos acceder a través del MCER, en función de las expectativas y necesidades como docentes.

${ }^{15}$ Propiedades seleccionadas para la categorización de los elementos de este estudio analítico, por su productividad como cualidades pragmáticas.
} 
comunicativas de la lengua- para su «legibilidad» primero, y para ser consultado y utilizado en una perspectiva de didáctica operativa.

b) Proponer las definiciones de dichos criterios, -en función de los índices específicos ${ }^{16}$ de ser: válidos, apropiados y realmente evaluables- para su «evaluabilidad» primero, y para ser jerarquizados, seleccionados y objeto de baremo de calificación según los contextos de aprendizaje, según las finalidades, etc. Todo ello conforme a las escalas A1, A2, B1, B2, C1, C2. (Ver ANEXO: Palabras / conceptos clave del estudio).

\subsection{Método de investigación: documentos y unidades de análisis, fases, procedimientos, estrategias y soportes}

Para el estudio analítico de estos dos documentos, hemos estimado la eficiencia y la utilidad del análisis de contenido que, en Bardin (1977) encontramos caracterizado como:

Un ensemble de techniques d'analyse des communications utilisant des procédures systématiques et objectives de description du contenu des messages (Bardin, 1977:38). Toute démarche qui, à partir d'un ensemble de techniques partielles mais complémentaires, consiste à expliciter et systématiser le contenu des messages et l'expression de ce contenu à l'aide d'indices quantifiables ou non. (Bardin, 1977:43).

Desde sus funciones heurísticas y verificadoras, sus procedimientos y técnicas nos han permitido - como recoge Legendre (1993: 45-46) - diseñar el proceso de investigación según el itinerario conceptual que ilustramos en el Cuadro 1:

Cuadro 1: Aspectos conceptuales para el proceso de investigación propiamente dicho

\begin{tabular}{|c|c|}
\hline $\begin{array}{c}\text { Procedimientos en el análisis de contenido } \\
\qquad \downarrow \downarrow\end{array}$ & $\begin{array}{c}\text { Itinerario conceptual del objeto de estudio } \\
\qquad \downarrow \downarrow\end{array}$ \\
\hline $\begin{array}{l}\text { Aislar la importancia cualitativa y cuantitativa } \\
\text { de algunos aspectos particulares de los } \\
\text { descriptores y las definiciones de objetivos } \\
\text { siendo éstos, pues, las unidades de análisis. }\end{array}$ & Elementos léxicos \\
\hline $\begin{array}{l}\text { Dar cuenta de las informaciones contenidas en } \\
\text { esas unidades de análisis, respecto al objeto de } \\
\text { estudio que nos ocupa (datos). }\end{array}$ & Indicadores de noción (Rey, 1992) que, \\
\hline Comprender e interpretar esos datos $\cdots$ & $\begin{array}{l}\text { por sus aspectos formales, axiológicos, } \\
\text { práxicos y explicativos puedan ser } \\
\text { identificados e interpretados como } \\
\vdots \\
\text { Criterios de evaluación. }\end{array}$ \\
\hline
\end{tabular}

${ }^{16}$ Índices que junto a las propiedades son el lugar de confluencia de los aspectos de orden formal, axiológico, práxico y explicativo que subyacen al establecimiento de los criterios de evaluación. 


\subsubsection{Unidades de análisis y unidades de registro}

Con una orientación prioritariamente cualitativa, para este proceso, pues:

a) Hemos tomado como material de estudio los dos referentes curriculares indicados para el ELE, al ser una fuente ineludible de información respecto al objeto que nos ocupa:

- el MCER (2002), (referencia para las concepciones sobre la enseñanza, el aprendizaje y la evaluación que nos ocupa); y

- el Plan Curricular del Instituto Cervantes (2006), (referencia para la elaboración de los programas de ELE).

b) Hemos delimitado como unidades de análisis - en el caso del MCER (2002)- los descriptores de los aspectos relativos a las competencias comunicativas de la lengua (MCER, 2002: 106-127) y a las actividades comunicativas de la lengua (MCER, 2002: 60-85), sobre los que se dan escalas ilustrativas tal y como indicamos en el Cuadro 2. Para este Cuadro queremos indicar también que los descriptores retenidos se relacionan de forma interdependiente y que entre ellos existen fuertes conexiones conceptuales, conforme hemos expuesto en el apartado 3, punto A):

Cuadro 2: Relación de los componentes de las competencias comunicativas de la lengua $y$ de las actividades que las actualizan. (Según el MCER, 2002: 106-127)

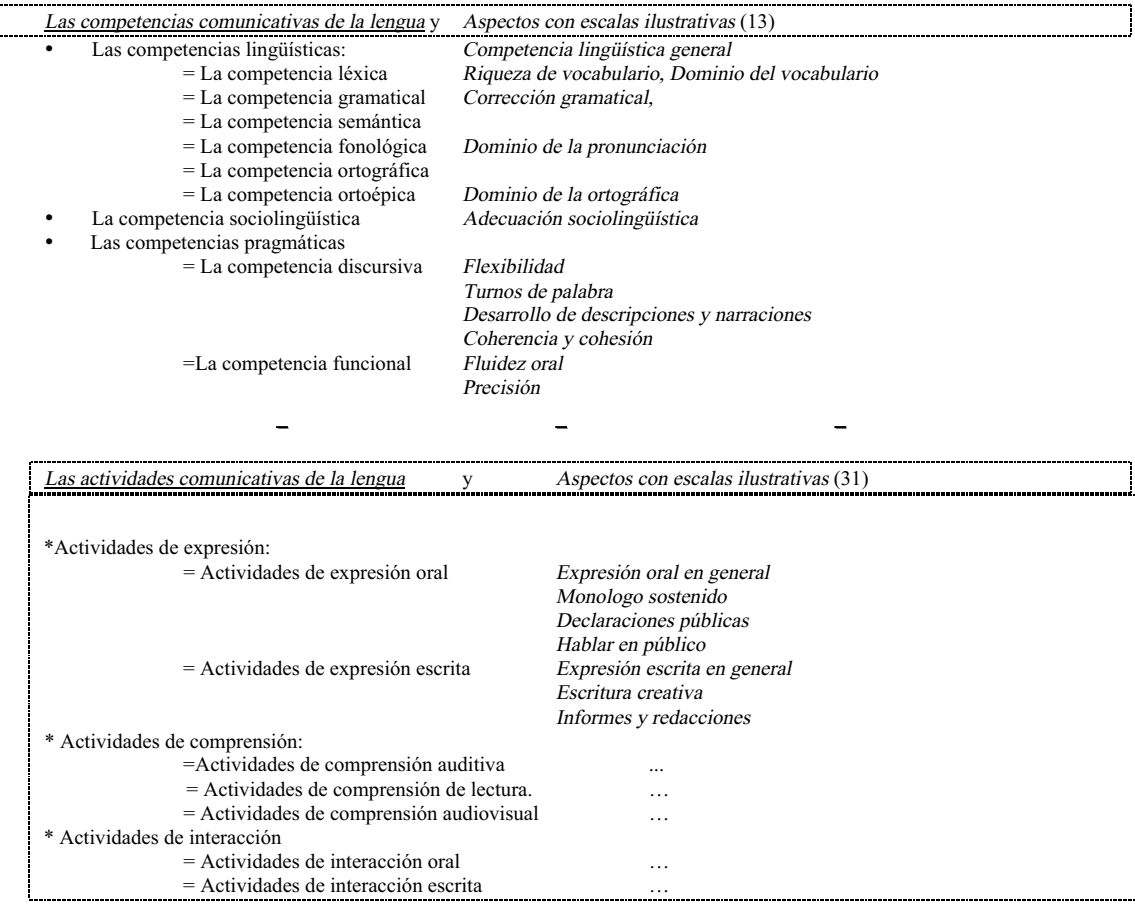


Son unidades de análisis - en el caso del Plan curricular del Instituto Cervantes (2006) - las definiciones de objetivos del apartado relativo a la dimensión: El alumno como agente social, y los textos de los apartados que los especifican relativos a: el desglose de sus aspectos, las características que subyacen a esos aspectos y la descripción general del nivel ${ }^{17}$. Estructura que ilustramos en el Cuadro 3:

Cuadro 3: Esquema de la estructura y organización de las definiciones de Objetivos generales (según el Plan Curricular del Instituto Cervantes, 2006: 79-82, vol. I: A1-A2)

\section{EI alumno como agente social}

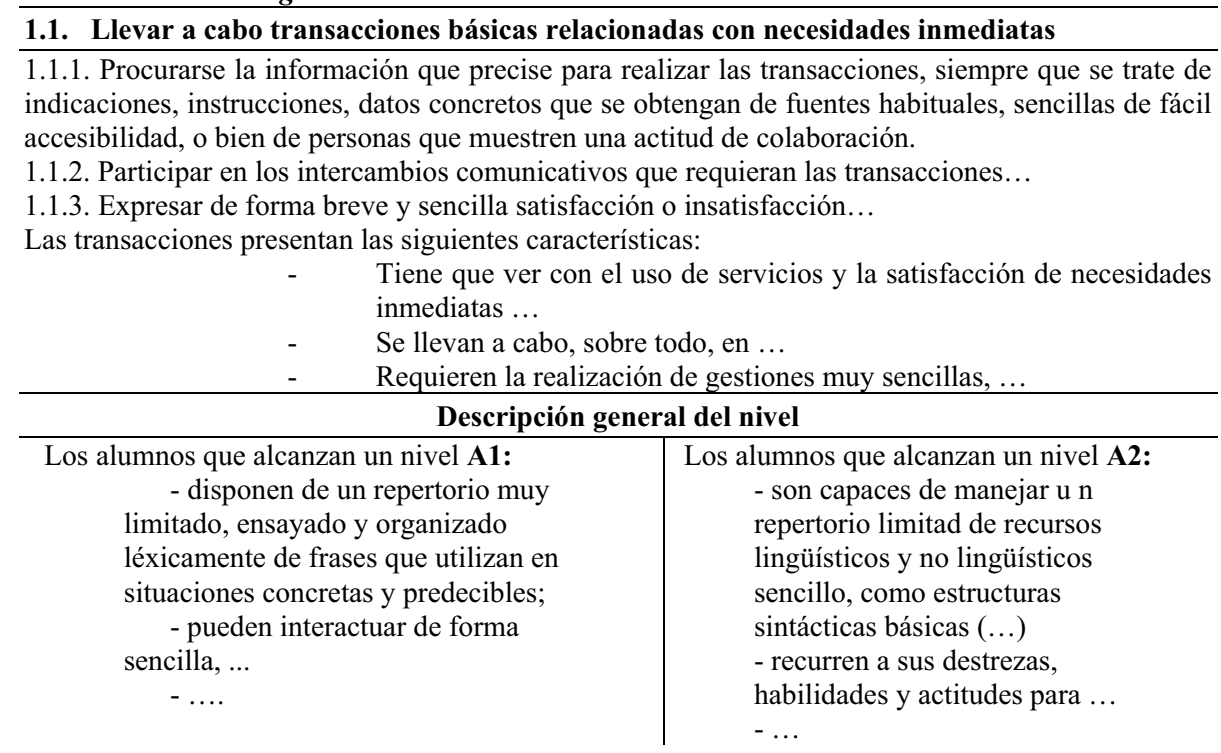

En uno y otro documento estudiados, son unidades de registro aquellos elementos léxi$\cos$ de tamaño y naturaleza variada que, como segmentos del contenido de los descriptores y de las definiciones de objetivos, son considerados en Rey (1992) indicadores de noción de criterio de evaluación.

Se trata de unidades de significación que proceden de una segmentación a nivel semántico en correspondencia con unidades formales, para ser categorizadas cualitativamente y consideradas, también, desde el punto de vista de su frecuencia de aparición. Todo ello considerando que -de la conjunción entre unidad de significación y unidad formal- deben resultar elementos léxicos marcados por las propiedades de ser transparentes, comunes y constantes o estables.

\footnotetext{
${ }^{17}$ A lo que se dedica el Capítulo 1, punto 1 en cada uno de los tres volúmenes del Plan curricular del Instituto Cervantes.
} 


\subsubsection{Fases, procedimientos, estrategias y soportes}

Hemos estructurado el proceso del análisis de contenido en dos grandes fases subsecuentes que, conforme a los objetivos formulados, han orientado la organización de las estrategias movilizadas y han determinado la configuración y los reajustes del dispositivo de análisis propiamente dicho. A saber:

- Fase 1 de observación, identificación-interpretación y registro.

- Fase 2 de clasificación, organización-descripción y definición.

Fase 1. Observación, identificación-interpretación y registro:

En esta Fase, hemos llevado a cabo una lectura previa de observación, para proceder en mejores condiciones a la identificación de aquellos elementos léxicos -indicadores de nociónque, interpretados como criterios de evaluación, se han constituido en unidades de base / registro.

Al tratarse de retener los elementos léxicos que son indicadores de noción de los aspectos - a priori más relevantes del uso de la lengua- como adelantamos más arriba:

a) Hemos partido de la consideración de aquellos aspectos cualitativos que, al respecto, establece el MCER en términos de: alcance, corrección, fluidez, coherencia, etc.

b) Hemos elaborado y utilizado nuestra categorización semántica adoptando una doble perspectiva para el tratamiento de los datos, cuyas informaciones se inscriban en el orden de lo formal, axiológico, práxico y explicativo. (Ver estos términos en ANEXO). Es decir que de forma complementaria:

b.1. Por una parte, hemos identificado-interpretado aquellos elementos léxicos que nos remiten a unidades de significación - núcleos de sentido- tales como características, grado, información normativa y práctica, cantidad, entre otros. Es decir, aquellos que traducen aspectos del orden de lo axiológico, lo práxico y lo explicativo.

b.2. Por otra parte, en la perspectiva de las unidades formales ${ }^{18}$, hemos retenido aquellos segmentos de contenido -elementos léxicos, indicadores de noción / criterios- cuyo número de unidades o elementos se estima óptimo para interpretar la significación ${ }^{19}$ (unidad de contexto) en su uso y aplicación, por cuanto que -en la perspectiva de Wittgenstein $(1953,1968)$ - es en ello en lo que reside, además, el significado de una expresión. Entendemos que así les dotamos de mayor precisión.

c) Hemos procedido a registrar los resultados de este conjunto de operaciones y acciones. Para ello, hemos diseñado soportes en forma de Parrillas que reproducimos a continuación:

- Las Parrillas 1a, para el inventario de los elementos léxicos estimados -conforme a lo expresado para esta Fase en el punto b.1- indicadores de noción/

\footnotetext{
${ }_{18}$ Del orden de lo formal.

${ }^{19}$ Consideración pues de la unidad de contexto dado que, en algunos casos, de la segmentación también depende la precisión de la significación.
} 
criterio $^{20}$, -por cada nivel y competencia comunicativa de la lengua primero. Podemos así poner de manifiesto las conexiones conceptuales entre los descriptores de esas competencias y los descriptores de las actividades comunicativas de la lengua en las que se encuentran las competencias.

\section{Parrilla 1a: Descriptores}

Nivel ...

Competencia ...

\begin{tabular}{|c|c|c|c|c|c|c|c|}
\hline $\begin{array}{l}\text { Indicador } \\
\text { de noción/ } \\
\text { criterio }\end{array}$ & $\begin{array}{l}\text { Expresión } \\
\text { oral }\end{array}$ & \begin{tabular}{|l} 
Expresión \\
escrita
\end{tabular} & $\begin{array}{l}\text { Comprensión } \\
\text { auditiva }\end{array}$ & $\begin{array}{l}\text { Comprensión } \\
\text { de lectura }\end{array}$ & $\begin{array}{l}\text { Comprensión } \\
\text { audiovisual }\end{array}$ & $\begin{array}{l}\text { Interacción } \\
\text { oral }\end{array}$ & $\begin{array}{l}\text { Interacción } \\
\text { escrita }\end{array}$ \\
\hline “...." & “...." & “...." & “...." & “...." & “...." & “...." & “...." \\
\hline
\end{tabular}

- Las Parrillas 1b, para el inventario de los elementos léxicos estimados -conforme a lo expresado para esta Fase en el punto b.1-indicadores de noción ${ }^{21}$ / criterio por cada nivel y definición de objetivo general. De la misma forma podemos así poner de manifiesto las conexiones conceptuales con las especificaciones sobre los aspectos de cada objetivo que tomamos como sub-indicadores de noción /criterio, con las características y con las descripciones generales del nivel.

Parrilla 1b: Objetivo(s) general(es)...

Nivel ...

El alumno como agente social

\begin{tabular}{|l|l|l|l|}
\hline $\begin{array}{l}\text { Indicador de } \\
\text { noción/ criterio }\end{array}$ & $\begin{array}{l}\text { Sub-indicadores de noción / } \\
\text { criterios }\end{array}$ & Características & $\begin{array}{l}\text { Descripción general del } \\
\text { nivel }\end{array}$ \\
\hline "..." & "..." & "..." & "..." \\
\hline
\end{tabular}

Fase 2. Clasificación, organización-descripción y definición:

a) Hemos establecido las conexiones conceptuales (campo conceptual) de los indicadores de noción / criterios retenidos, dentro de cada escala (niveles de referencia A1, A2, B1, B2, C1, C2), conducentes a clasificar los indicadores de noción / criterios retenidos conforme presenten las propiedades de ser transparentes, comunes y constantes o estables, de forma transversal a las unidades de análisis descritas.

En un primer momento, dichas conexiones conceptuales han sido efectuadas de forma individual por cada miembro del equipo; en un segundo momento, en sesiones colectivas para, finalmente, verificar los resultados contrastando muestras significativas de nuestro análisis en las consultas a profesores de lenguas.

${ }^{20}$ Conforme a las categorías de análisis establecidas y definidas en el ANEXO.

${ }^{21}$ Conforme a las categorías de análisis indicadas y definidas en el ANEXO. 
b) Hemos procedido a organizar y describir los datos, efectuando las operaciones complementarias siguientes:

b.1) combinación y asociación;

b.2) reformulación, y la operación subsecuente

b.3) reducción, en las que como plantean Huberman y Miles (1991):

La condensation des données s'opère continuellement dans toutes les phases d' un projet à orientation qualitative ...

...consiste à élaguer, trier, distinguer, rejeter et organiser les données de telle sorte qu'on puisse en tirer des conclusions «finales » et les vérifier. (Huberman y Miles 1991: 35).

Para b.1.) Combinación y asociación, hemos trabajado por equivalencias, similitudes y contigüidades percibidas en las significaciones -por núcleos de sentido- y reconocidas y consensuadas por los miembros del equipo y los profesores consultados, con el fin de retener aquellos que son comunes, constantes o estables.

Para b.2) Reformulación, hemos propuesto aquellos enunciados de los criterios de evaluación en términos que sean transparentes (para su legibilidad).

Para b.3) Reducción, hemos atendido a las consideraciones de base de los especialistas en materia de evaluación, en el sentido de que:

...cualquier sistema práctico de evaluación tiene que reducir el número de categorías posibles a un número viable.

La experiencia demuestra que más de cuatro o cinco categorías comienzan a provocar una sobrecarga cognitiva... (MCER, 2002: 193).

...un conjunto más pequeño de criterios de evaluación que sea adecuado para las necesidades de los alumnos, para los requisitos de la tarea de evaluación concreta y para el estilo de cultura pedagógica. (MCER, 2002: 194).

Lo cual da lugar a fijar y determinar de forma más precisa los criterios de evaluación esenciales por su condición de indispensables; es decir, aquellos con los índices específicos de ser válidos, apropiados y realmente evaluables.

Al término de estas operaciones y acciones, obtenemos un repertorio descriptivo de criterios de evaluación que tomamos, a su vez, como base para la elaboración de un repertorio de criterios de evaluación -definidos con carácter estructural u operativo y analítico- que contribuya a aportar sobre ellos mayores precisiones. Se trata de definiciones de carácter estructural u operativo, por cuanto que se da cuenta de sus componentes y relaciones particulares posibles. Y, de carácter analítico por cuanto que las definiciones propuestas se apoyan en otros indicadores de noción retenidos (Legendre, 1993).

Los resultados de este tratamiento de los datos son formalizados en aquellos soportes que se han diseñado en forma de Tablas y cuyos tipos dependen de la orientación y contenido del repertorio. Para cada nivel disponemos de:

- Tablas para el Repertorio descriptivo A): Repertorio descriptivo A.1a)

Repertorio descriptivo A.1b)

- Tablas para el Repertorio descriptivo B): Repertorio descriptivo B.1a)

- Tablas para el Repertorio C) Definiciones 
- El tipo de tabla correspondiente al Repertorio descriptivo A.1a) de criterios de evaluación relativos a cada competencia y para cada nivel, y al Repertorio descriptivo A.1b) de criterios de evaluación relativos a cada objetivo general y para cada nivel, se ha diseñado con el formato que reproducimos a continuación. En estos repertorios se ofrecen los resultados intermedios de las sucesivas operaciones de combinación y reformulación efectuadas sobre los datos de las respectivas Parrillas 1a y 1b. para cada competencia y objetivo general. Damos cuenta de la frecuencia de aparición y de las propiedades asociadas más estrictamente que nos permitan tomar las decisiones oportunas en la siguiente operación de reducción, así como organizar las definiciones.

Repertorio descriptivo A.1a)

\begin{tabular}{|c|c|c|c|}
\hline \multicolumn{4}{|c|}{$\begin{array}{l}\text { CRITERIOS DE EVALUACIÓN } \\
\text { COMPETENCIA.... } \\
\text { NIVEL ... }\end{array}$} \\
\hline $\begin{array}{l}\text { Indicador de } \\
\text { noción / criterio }\end{array}$ & $\begin{array}{c}\text { Propiedades: } \\
\text { comunes y constantes o estables }\end{array}$ & $\begin{array}{l}\text { Propiedades: } \\
\text { transparentes }\end{array}$ & Otros \\
\hline$\ldots$ & $\ldots$ & $\cdots$ & $\ldots$ \\
\hline
\end{tabular}

Repertorio descriptivo A.1b)

\begin{tabular}{|c|c|c|c|}
\hline \multicolumn{4}{|c|}{$\begin{array}{l}\text { CRITERIOS DE EVALUACIÓN } \\
\text { OBJETIVO GENERAL.... } \\
\text { NIVEL ... }\end{array}$} \\
\hline $\begin{array}{l}\text { Indicador de } \\
\text { noción / criterio }\end{array}$ & $\begin{array}{c}\text { Propiedades: } \\
\text { comunes y constantes o estables }\end{array}$ & $\begin{array}{l}\text { Propiedades: } \\
\text { transparentes }\end{array}$ & Otros \\
\hline$\ldots$ & $\ldots$ & $\cdots$ & $\ldots$ \\
\hline
\end{tabular}

- El tipo de tabla correspondiente al Repertorio descriptivo B.1a) y al Repertorio descriptivo B.1b) de criterios de evaluación, se ha diseñado con el formato que reproducimos a continuación, para dar cuenta de los resultados de la operación de reducción sobre los datos de los respectivos Repertorios descriptivos A.1a) y A.1b). Igualmente se elaboran estas tablas por niveles y ofrecen los resultados intermedios para cada competencia y objetivo general. Se pretende que los criterios retenidos estén asociados, así, a los índices más específicos de ser válidos, apropiados y evaluables. 


\section{Repertorio descriptivo B.1a)}

\begin{tabular}{|c|}
\hline $\begin{array}{l}\text { CRITERIOS DE EVALUACIÓN } \\
\text { COMPETENCIA ... } \\
\text { NIVEL ... }\end{array}$ \\
\hline Indicador de noción / criterio \\
\hline \\
\hline
\end{tabular}

Repertorio descriptivo B.1b)

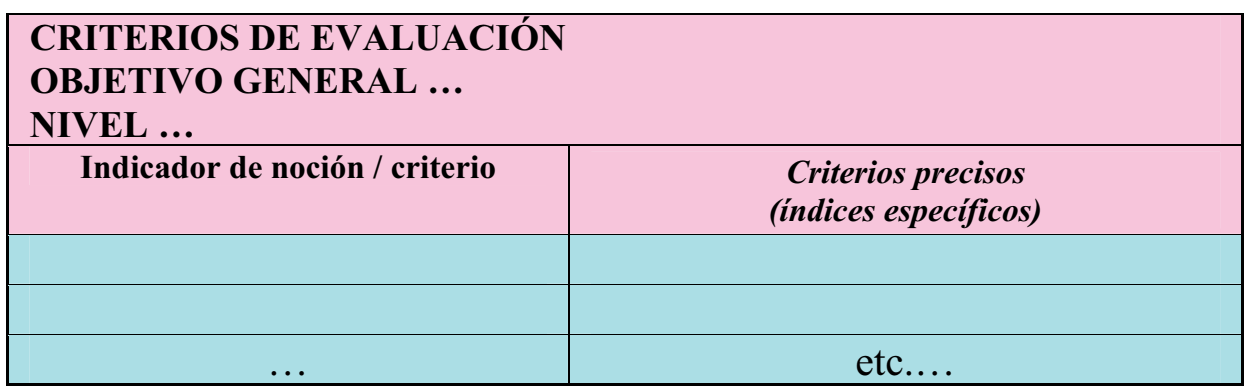

- El tipo de tabla correspondiente al Repertorio C) Definiciones de los criterios de evaluación finalmente da cuenta -de forma afirmativa- de la significación más precisa del criterio.

Como corresponde en el orden propiamente lingüístico de toda definición está dotada de una formula explicativa, a la que conferimos - en una perspectiva de didáctica operativa tal como hemos expuesto más arriba - de un carácter tanto operativo como analítico ya que hemos pretendido que de cuenta de sus componentes, apoyándose en otros elementos léxicos (indicadores de noción / criterios) interrelacionados.

\section{Los datos Y RESUltados del ANÁlisis APLiCAdo A LA COMPETENCIA LIN- gǘstica GeNeral Relativos al Nivel C2}

A título ilustrativo, exponemos el análisis de contenido que se inicia partiendo de los descriptores de la competencia lingüística general (MCER, 2002: 107) la cual, en la descripción de las competencias lingüísticas, se contempla como uno de los aspectos sobre los que se dan escalas ilustrativas (según hemos expuesto en 3.2.1).

Ofrecemos el tratamiento de los datos del Nivel C2 por ser éste muy productivo en el sentido de que, especialmente en los descriptores correspondientes a las actividades comunicativas de la lengua, al remitirnos en varios casos a los descriptores de los niveles C1 (en 10 casos), 
B2 (en 5 casos) e incluso a B1 (en un caso $^{22}$ ), obtendremos datos para varios niveles simultáneamente. Solo en 2 casos se nos indica que no hay descriptor disponible ${ }^{23}$.

Por lo que se refiere a la Parrilla 1a), en la Fase 1, los elementos léxicos que hemos registrado como indicadores de noción /criterios de evaluación son aquellos que recogen las informaciones que, prioritariamente, nos remiten a los aspectos de: características, grado, información normativa y práctica, cantidad, entre otras.

Así es como las unidades de contexto (según hemos expuesto en punto b.2, apartado 3.2.2.) aportan precisiones tales como:

- discursos, descripciones, temas, preguntas, textos, historias, conversaciones, informes, redacciones, descripciones, etc.; (características)

- alto grado de, muy, buen, etc.; (grado)

- con seguridad, con éxito, apropiado, eficaz, etc.; (información normativa y práctica)

- amplia gama, cantidad considerable de, etc. (cantidad).

\footnotetext{
${ }^{22}$ Ver que es el caso de las actividades de interacción escrita, relativas a notas mensajes y formularios (MCER, 2006: 82).

${ }^{23}$ En las actividades de expresión oral, se trata del caso relativo a: monólogo sostenido: argumentación y del caso relativo a: declaraciones públicas.
} 


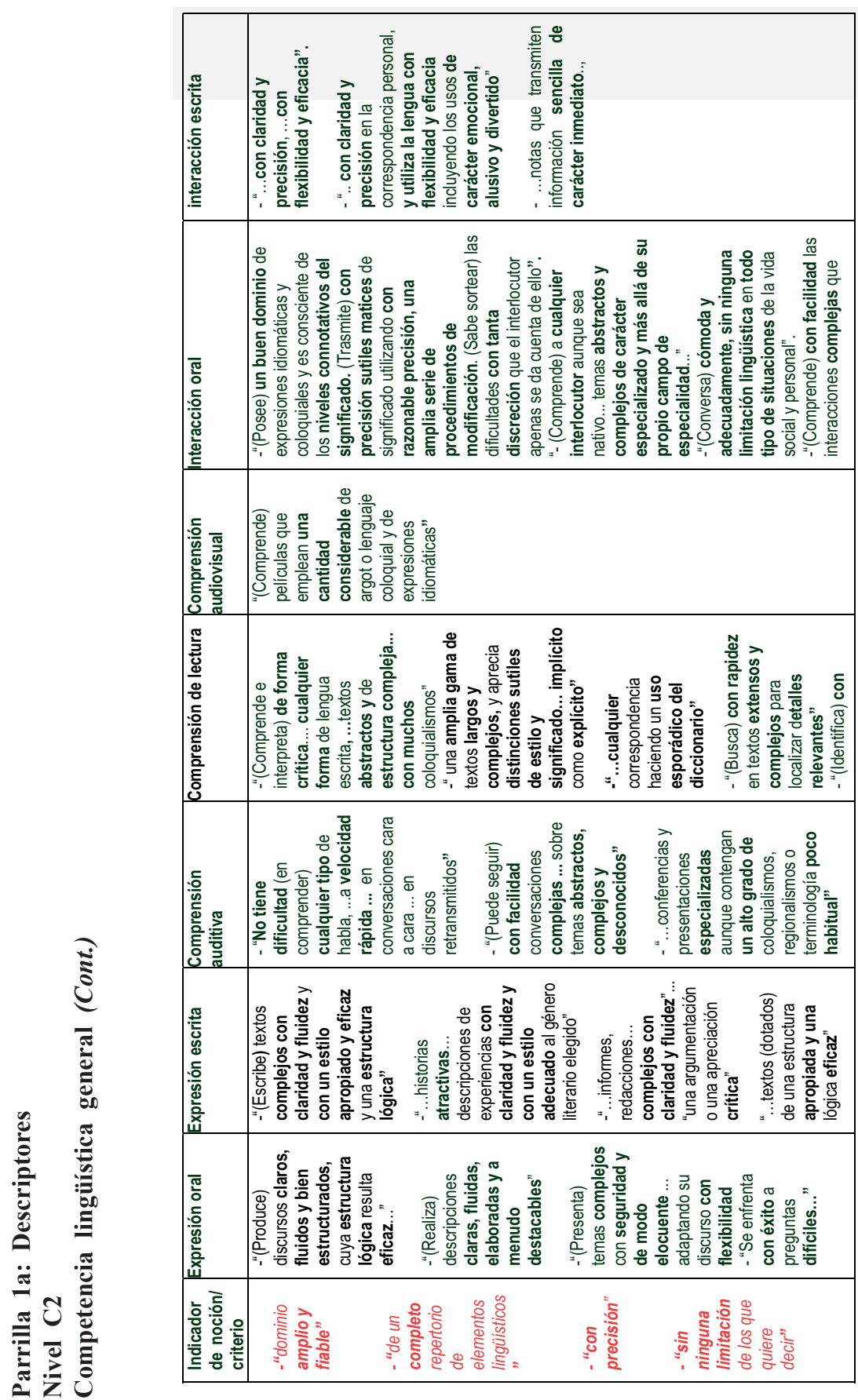




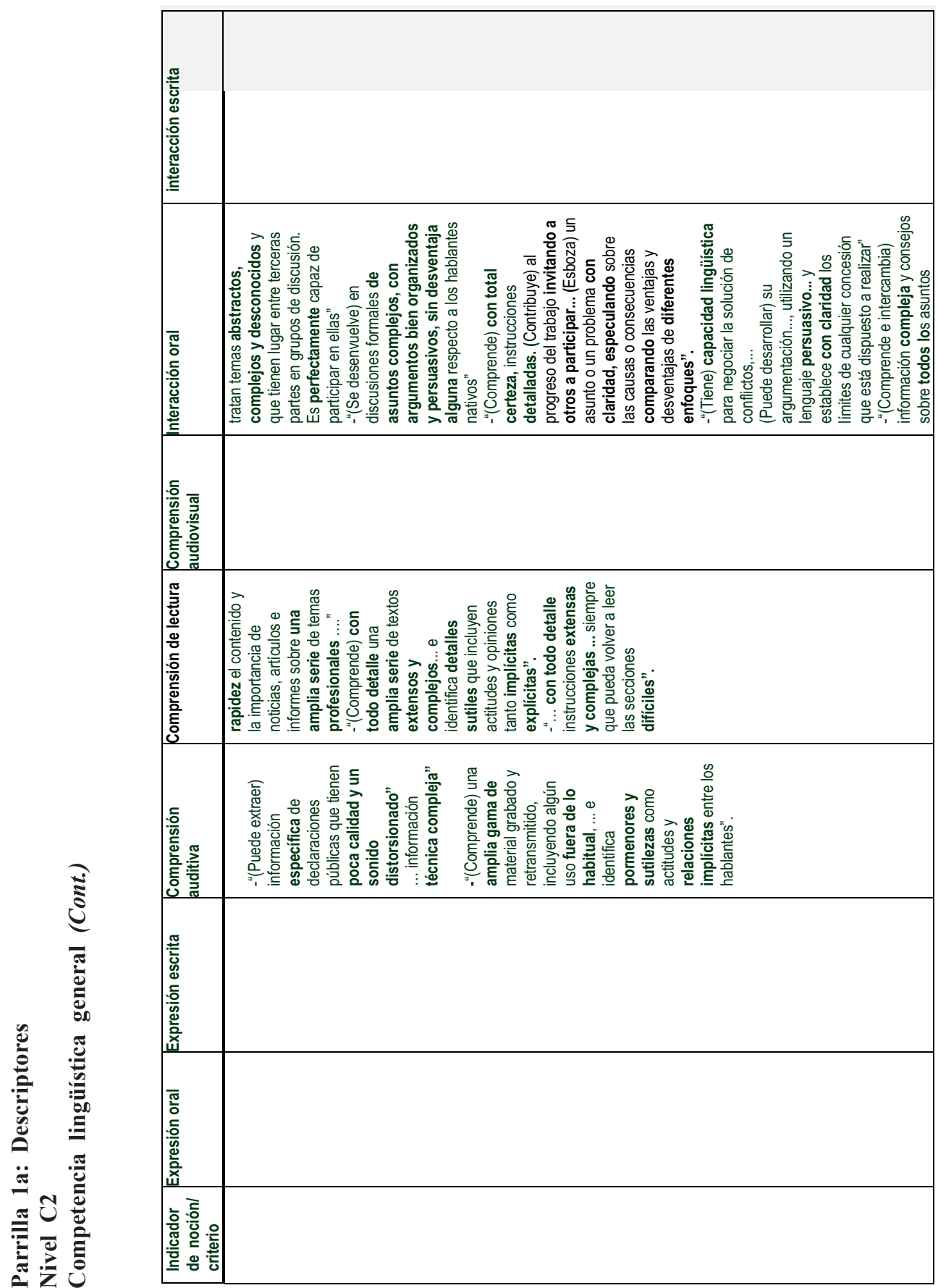




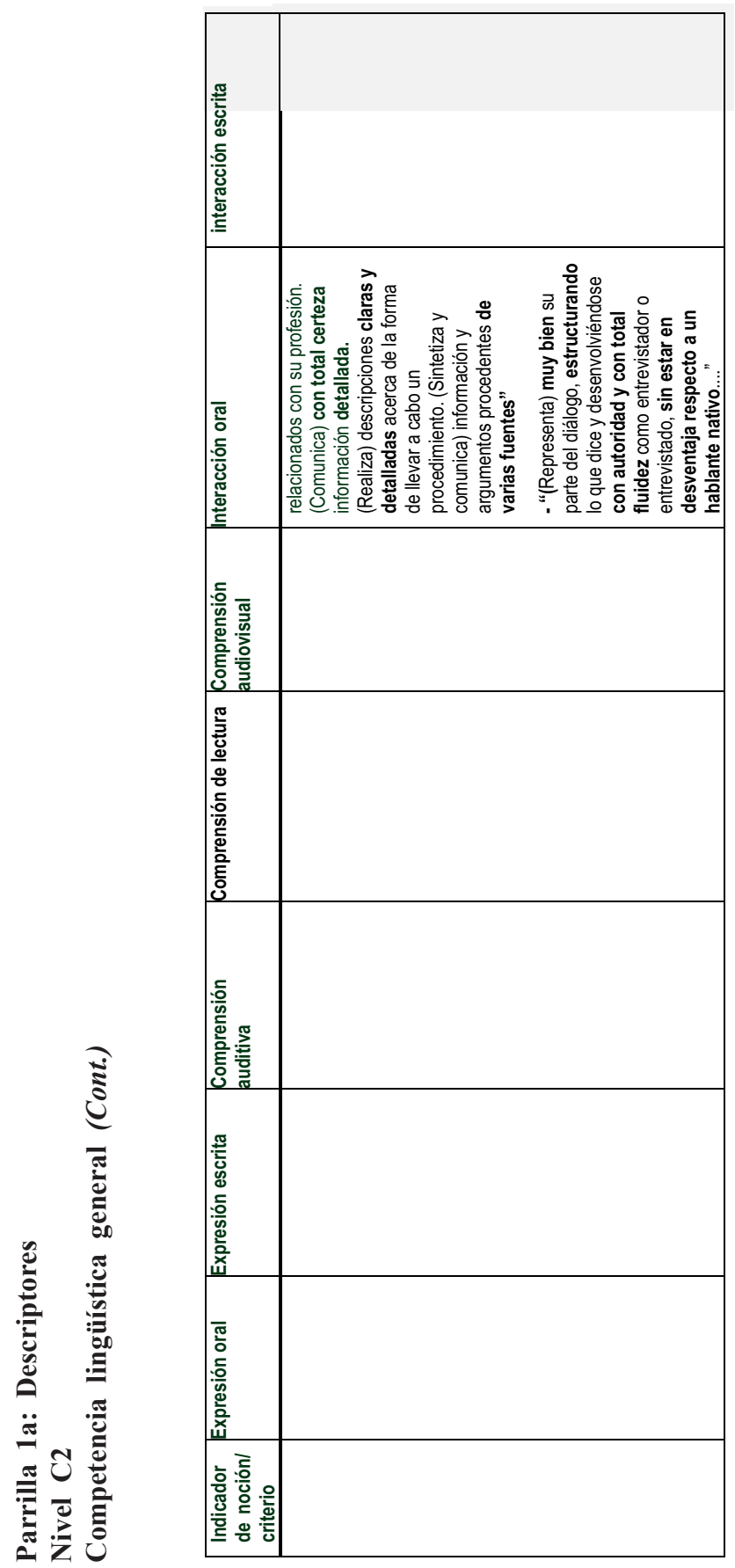


Por lo que se refiere a la Fase 2 del proceso de análisis para los datos de la Parrilla 1a), los procedimientos para las conexiones conceptuales han permitido clasificar los indicadores de noción / criterios conforme cuya organización y descripción recoge la Tabla correspondiente al Repertorio descriptivo A.1a), de forma integrada junto a la frecuencia de aparición. Hemos realizado:

- por una parte, las combinaciones y asociaciones por similitudes y contigüidades,

- por otra parte, las reformulaciones.

\section{Repertorio descriptivo A.1a)}

\begin{tabular}{|c|c|c|c|}
\hline \multicolumn{4}{|c|}{$\begin{array}{l}\text { CRITERIOS DE EVALUACIÓN } \\
\text { COMPETENCIA LINGÜÍSTICA GENERAL } \\
\text { NIVEL C2 }\end{array}$} \\
\hline $\begin{array}{l}\text { Indicador de } \\
\text { noción / } \\
\text { criterio }\end{array}$ & $\begin{array}{c}\text { Propiedades: } \\
\text { comunes y constantes } 0 \text { estables }\end{array}$ & $\begin{array}{l}\text { Propiedades: } \\
\text { transparentes }\end{array}$ & Otros \\
\hline $\begin{array}{l}\text { "(dominio) } \\
\text { amplio" } \\
\text { "un completo } \\
\text { (repertorio de } \\
\text { elementos } \\
\text { lingüísticos)" }\end{array}$ & $\begin{array}{l}\text { ๑-complejo (16) } \\
\text { *-largos /extensos (4) } \\
\text { *-profesionales } \\
\text { o-abstracto (4) } \\
\text { o-desconocido (2) } \\
\text { o-poco / fuera de lo habitual (2) } \\
\text { o-difícil (3) } \\
\text { \#-mas allá de su (propio campo de) } \\
\text { especialidad }\end{array}$ & $\begin{array}{l}\diamond \text {-estructura / técnica compleja } \\
(2) \\
\text { *-con un alto grado de } \\
\text { *-amplia gama / serie de (5) } \\
\text { *-con muchos } \\
\text { *-sin ninguna limitación } \\
\text { lingüística } \\
\text { *-una cantidad considerable de } \\
\text { o-especializado (4) } \\
\text { \#-cualquier / cualquier forma / } \\
\text { tipo de (4) } \\
\text { \#-todos / todo tipo de (2) } \\
\text { \#-diferentes enfoques } \\
\text { \#-varias fuentes }\end{array}$ & $\begin{array}{l}\text { - (historias) } \\
\text { atractivas } \\
\text {-en conversaciones / } \\
\text { retransmitido } \\
\text { cara a cara } \\
\text {-uso esporádico } \\
\text { del diccionario } \\
\text {-poca calidad } \\
\text {-sonido } \\
\text { distorsionado }\end{array}$ \\
\hline $\begin{array}{l}\text { "(dominio) } \\
\text { fiable" }\end{array}$ & $\begin{array}{l}\diamond \text {-claro(5) } \\
\text { *-fluido (2) } \\
\text { *-destacable /buen dominio de (2) } \\
\text { o-apropiado (2) } \\
\text { o-adecuado } \\
\text { o-(muy) bien organizado (2) } \\
\text { \#-eficaz (4) }\end{array}$ & 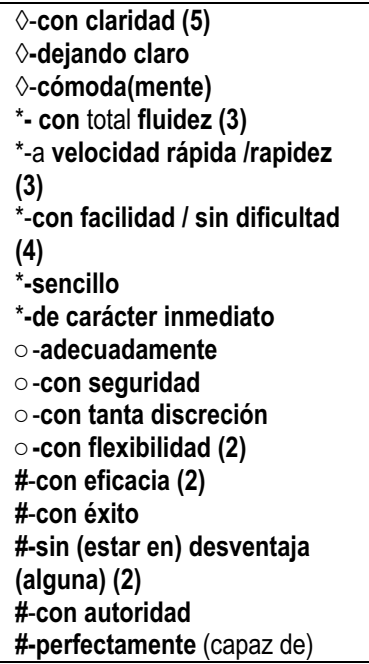 & $\begin{array}{l}\text {-persuasivo (2) } \\
\text {-de modo } \\
\text { elocuente } \\
\text {-especulando } \\
\text {-comparando } \\
\text {-adaptando }\end{array}$ \\
\hline
\end{tabular}


Repertorio descriptivo A.1a) (Cont.)

\begin{tabular}{|c|c|c|c|}
\hline $\begin{array}{l}\text { "con } \\
\text { precisión" }\end{array}$ & $\begin{array}{l}\diamond \text {-bien estructurado } \\
\diamond \text {-estructura lógica(2) } \\
\diamond \text {-relaciones (implícitas) } \\
\text { *-detalladas (4) } \\
\text { *- explícito (3) } \\
\text { *-detalles relevantes (2) } \\
\text { *-precisiones } \\
\text { o-niveles connotativos } \\
\text { o-(información) específica } \\
\text { o- implícito (3) } \\
\text { \#-sutileza /-sutiles matices (3) }\end{array}$ & $\begin{array}{l}\diamond \text {-estructurando } \\
\diamond \text {-elaborado } \\
*_{\text {-con todo detalle }(2)} \\
{ }^{*} \text {-pormenores } \\
{ }^{*} \text {-con (razonable) precisión (4) } \\
\text { o-con total certeza (2) } \\
\text { \#sutilezas/ } \\
\text { distinciones sutiles (2) }\end{array}$ & $\begin{array}{l}\text {-(apreciación) } \\
\text { crítica I } \\
\text {-de forma crítica } \\
\text {-de carácter } \\
\text { emocional, alusivo, } \\
\text { divertido }\end{array}$ \\
\hline
\end{tabular}

Igualmente ilustramos el resultado de la operación de reducción, en la Tabla correspondiente del Registro descriptivo B.1a), para la que hemos obtenido los siguientes datos como base para el Repertorio C: Definiciones, tal y como ilustramos a continuación.

\section{Repertorio descriptivo B.1a)}

\begin{tabular}{|c|c|}
\hline \multicolumn{2}{|c|}{$\begin{array}{l}\text { CRITERIOS DE EVALUACIÓN } \\
\text { COMPETENCIA LINGÜÍSTICA GENERAL } \\
\text { NIVEL C2 }\end{array}$} \\
\hline Indicador de noción / criterio & $\begin{array}{l}\text { Criterios precisos } \\
\text { (índices especificos) }\end{array}$ \\
\hline - AMPLIO / COMPLETO & $\begin{array}{l}\text {-complejo (18) } \\
\text { *-extenso (14) } \\
\text { o-difícil (15) } \\
\text { \#-variado }(9)\end{array}$ \\
\hline - FIABLE & $\begin{array}{l}\text { O-claro (12) } \\
\text { *-fluido }(16) \\
\text { o-apropiado }(10) \\
\text { \#-eficaz }(11) \\
\end{array}$ \\
\hline - CON PRECISIÓN & $\begin{array}{l}\text {-estructurado (6) } \\
\text { *-detallado (17) } \\
\text { o-acertado (7) } \\
\text { \#-sutil (5) }\end{array}$ \\
\hline
\end{tabular}

Repertorio C): Definiciones

\begin{tabular}{|c|c|}
\hline \multicolumn{2}{|c|}{$\begin{array}{l}\text { CRITERIOS DE EVALUACIÓN } \\
\text { COMPETENCIA LINGÜIISTICA GENERAL } \\
\text { NIVEL C2 }\end{array}$} \\
\hline - $\frac{\text { AMPLIO Y/O }}{\text { COMPLETO }}$ & $\begin{array}{l}\text { corresponde a la noción de "sin límites en algunos aspectos", de lo cual } \\
\text { da cuenta (y puede ser evaluado a través de): } \\
\text { lo complejo, lo extenso, lo difícil y lo variado. }\end{array}$ \\
\hline - FIABLE & $\begin{array}{l}\text { corresponde a la noción de "buen funcionamiento del uso de la } \\
\text { lengua", de lo cual da cuenta (y puede ser evaluado a través de): } \\
\text { lo claro, lo fluido, lo apropiado y lo eficaz. }\end{array}$ \\
\hline - CON PRECISIÓN & $\begin{array}{l}\text { corresponde a la noción "seguridad y exactitud en la ejecución", de lo } \\
\text { cual da cuenta (y puede ser evaluado a través de): } \\
\text { lo estructurado, lo detallado, lo acertado y lo sutil. }\end{array}$ \\
\hline
\end{tabular}




\section{REFERENCIAS BIBLIOGRÁFICAS}

Bardin, L. (1977). L'analyse de contenu. Paris : Presses Universitaires Françaises.

Bourguignon, C.; Delahaye, Ph. et Puren, C. (2007). Évaluer dans une perspective actionnelle. Le Diplôme de Compétence en Langue. Le Havre : Editions Belbopur.

Delorme, Ch. (dir.) (1994). L'évaluation en questions. Paris : ESF éditeur (5 ed.).

García Doval, F.; Guillén, C.; González Piñeiro, M.; González Porto, J.; Serna, I. y Vez, J. M. (2004). «Marco y Portafolio: porta linguarum para los europeos», en Porta Linguarum. Revista Internacional de Didáctica de las Lenguas Extranjeras, 2: 69-92.

Guide pour l'élaboration des politiques linguistiques éducatives en Europe. De la diversité linguistique à l'éducation plurilingue (2007). Conseil de L'Europe : Division des Politiques Linguistiques.

Guillén Díaz, C. (2005). «Para una 'cultura conceptual común` sobre los términos clave del Marco, Portfolio y Manual», en Glosas Didácticas, 14: 22-36.

Guillén, C.; Blasco, T. y Calleja, I. (1998). «La evaluación de la competencia comunicativa en las áreas de lengua. Análisis de las concepciones y actuaciones docentes», en Comunicación y Educación (C\&E), 9: 97-111.

Guillén, Díaz, C.; Santos, Ma J.; Ibáñez, J. y Sanz, E. (2007). «Los rasgos de la atención lingüística a los inmigrantes en Castilla y León y el marco de la política lingüística educativa en Europa», en Migraciones y desarrollo humano. V Congreso sobre la Inmigración en España. Mesa 5. Educación, Escuela, Familia y Juventud en la Inmigración. www.adeit.uv.es/ inmigracion 2007

Huberman, A. M. y Miles, M.B. (1991). Analyse des données qualitatives. Recueil de nouvelles méthodes. Bruxelles : De Boeck-Wesmael. S. A.

Ketele (de) J. M. (1986). L'évaluation: approche descriptive ou prescriptive. Bruxelles : De Boeck. Legendre, R. (1993). Dictionnaire actuel de L'Éducation. Montréal : Guérin / Paris : Eska.

Lussier, D. et Turner, C. E. (1995). Le Point sur l'évaluation en didactique des langues. Montréal : CEC.

MCER (2002). Marco común europeo de referencia para las lenguas: aprendizaje, enseñanza, evaluación. Madrid: Ministerio de Educación Cultura y Deporte / Anaya / Consejo de Europa / Instituto Cervantes (para la edición impresa en español).

Milanovic, M. (2002). Évaluation de compétences en langues et conception de tests. Strasbourg: Division de Politiques Linguistiques. (A.L.T.E.)

Montes Mozo, C. (2005). «La evaluación de lenguas extranjeras según las iniciativas y propuestas del Marco común europeo de referencia» en C. Montes Mozo (dir.). La enseñanza de lenguas desde una perspectiva europea. Madrid: Ministerio de Educación y Ciencia. Secretaría General Técnica, 159-180.

North, B. (1996). The Development of a Commun Framework Scale of Language Proficiency. New York: Peter Lang / PhD Thesis, Thames Valley University.

Parrondo Rodríguez, J. R. (2004). «Modelos, tipos y escalas de evaluación» en J. Sánchez Lobato e I. Santos Gargallo (dirs.), Vademécum para la formación de profesores. Enseñar español como segunda lengua (L2)/lengua extranjera (LE). Madrid: SGEL, 967-982.

Plan curricular de Instituto Cervantes (2006). Niveles de referencia para el español. Vol.I: A1-A2; Vol.II: B1-B2; Vol. III: C1-C2. Madrid: Instituto Cervantes / Biblioteca Nueva.

Puren, C. (2001). «La problématique de l'évaluation en didactique scolaire », en Les Langues Modernes, 2: 12-29. 
Raynal, F. y Rieunier, A. (1997). Pédagogie: dictionnaire des concepts clés. Apprentissages, formation, psychologie cognitive. Paris : ESF éditeur.Recommandation (2006/926/CE). «Recommandation du Parlement Européen et du Conseil, du 18 décembre 2006 sur les compétences clés pour l'éducation et la formation tout au long de la vie », en Journal officiel de L'Union européenne, 394 : 10-18. (du 30.12.2006).

Rey, A. (1992). La terminologie. Noms et notions. Paris: PUF.

Roy, D. (1991).Étude de l'importance des connaissances de l'enseignant et de l'influence des actes professionnels d'enseignement sur l'apprentissage collégial. Québec: Bibliothèque nationale du Québec 7 Cégep de Rimouski. Service de recherche et perfectionnement.

Tagliante, Ch. (2005). L'évaluation et le Cadre européen commun. Paris : Clé International (nouvelle édition) 


\section{ANEXO: Palabras / conceptos Clave del estudio}

Elemento léxico retenido: Aquella palabra o grupos de palabras que funcionen como «indicador de noción» / concepto que, por su significación primero e índices y propiedades, designe a un criterio de evaluación.

Indicador de noción: Signo aparente y probable que indica la existencia de una característica, un grado, una información normativa y práctica, cantidad, entre otras, respecto al uso de la lengua.

Criterio: Signo de un objetivo que permite distinguir entre personas, cosas o conceptos. Cualidad o norma que permite aplicar un juicio. Es un principio o razonamiento que permite aplicar un juicio de valor y fundamentar una decisión.

Unidades de análisis: Son los «segmentos de información» que para nosotros constituyen en este caso los enunciados de los descriptores del MCER y, cuando nos refiramos al Plan curricular del Instituto Cervantes los enunciados de los objetivos.

Aspectos (del orden de lo) formal, axiológico, práxico y explicativo de los elementos léxicos (puede ocurrir que más de uno de estos aspectos estén presentes en un elemento léxico retenido):

- formal, como manera «reconocible» de presentarse, de ser expresado.

- axiológico, del orden de los valores y las finalidades de la e/a de las lenguas en este caso.

- práxico, relativo a la manera concreta de realizar, actuar, (de aplicar los conocimientos) en la práctica, en las realizaciones en/de las actividades comunicativas de la lengua.

- explicativo, que hace claro y comprensible (el objetivo) y en el caso que nos ocupa el criterio, le hace comprender, da detalles, justifica, etc... (partiendo de lo axiológico, podríamos decir que es «del orden de las razones»).

- conexiones conceptuales, como operaciones de organización de los indicadores de noción retenidos, porque estén asociados al concepto etiqueta: criterio de evaluación. En su conjunto, los repertorios de esos indicadores de noción retenidos constituyen un campo conceptual.

(Estos aspectos se cruzan con las «categorías de análisis» en las operaciones de identificación e interpretación- subyacentes).

transparentes, que presenten claramente la orientación del juicio de valor que se debe emitir.

comunes, que concierne / aparece en la mayoría de los descriptores (de los de competencias, de los de actividades comunicativas de la lengua así como en las definiciones de los objetivos generales)

constantes o estables que no cambia (en la mayoría de los aspectos formal, axiológico, práxico $y$ explicativo de cada elemento léxico retenido)

válidos, que tienen las formas y condiciones para ser reconocido y aceptado, que es admisible, que tiene o se le puede atribuir un valor.

apropiados (como convenientes), que son propio de las realizaciones comunicativas de la lengua. (por ejemplo: una realización lingüística puede ser clara, breve, correcta....).

evaluables, sobre los que se pueda aplicar / determinar un juicio de valor / una cantidad. 